\title{
Incidence and Public Health Significance of Yersinia enterocolitica in Enugu Urban-Enugu State,Nigeria.
}

\author{
Mbah Modesta Ifeoma \\ Department Of Applied Microbiology And Brewing Nnamdi Azikiwe University,Awka,Anambra state ,Nigeria.
}

\begin{abstract}
Yersinia enterocolitica in animals in Enugu Urban- Enugu State, Nigeria.

A total of 106 samples, comprising 50 chickens and 33 goats from Artisan market and 23 pigs from New market,all in Enugu urban, were examined for the presence of Yersinia enterocolitica .Cold enrichment in a phosphate buffered saline, culture and biochemical tests were used as the diagnostic laboratory tests. This includes incubating the swabbed sample in freshly prepared $0.5 \% \mathbf{K O H}$ in $0.5 \% \mathrm{NaCl}(\mathrm{pH} 7.6)$ at $4^{\circ} \mathrm{C}$ for 3 weeks followed by culturing on nutrient agar at $37^{\circ} \mathrm{C}$ for $24 \mathrm{~h}$ and then on MacConkey agar at $37^{\circ} \mathrm{C}$ for $24 \mathrm{~h} . \mathrm{A}$ total of 5 Yersinia enterocoiltica were obtained- two were found in goats, one was found in chicken and 2 were found in pigs. Prevalence rates of $6.06 \%, 8.7 \%$ and $2 \%$ were found in goats, pigs and chickens, respectively .The prevalence rate of the different animals sampled combined was $4.72 \%$. This confirmed that pigs had the highest prevalence of $Y$. enterocolitica among the animals used for the study. Also the public health significance and recommendations to be followed in the processing and handling, of animals especially their chitterlings were discussed.

Key words: Prevalence rate, public health significance, cold enrichment, Yersinia enterocolitica biochemical tests.
\end{abstract}

\section{Introduction}

Yersinia enterocolitica is a species of gram-negative coccobacillus-shaped bacteria. It belongs to the family Enterobacteriaceae. $Y$. enterocolitica causes the disease yersiniosis. Yersiniosis is a zoonotic disease occurring in humans as well as wide array of animals such as cattle, deer, pigs, goats, birds. Many of these animals recover from the disease and become assymptomatic carriers (Collins, 1996). It infects the host by sticking to the cells of the host using Trimeric Autotransporter Adheesins (TAA) Yersinia enterocolitica is a non lactose fermenting gram negative rod that is urease positive and oxidase negative. It ferments glucose. The presence of bile salts in MacConkey agar prevents the organisms ability to ferment lactose. $Y$. enterocolitica does not produce hydrogen sulfide in tripe iron medium. (Brooks et al, 2005). It demonstrates significant morphologic pleomorphism. The organism is a facultative anaerobe that is motile at $25^{\circ} \mathrm{C}$ and non motile at $37^{\circ} \mathrm{C}$ (Brooks ,et al. 2005).

$Y$. enterocolitica strains and related species can be separated serologically into groups based on their heat-stable somatic antigens. Wauters describe 54 serogroups for $Y$. enterocolitica and related species. Aleksie and Bockemuhl, 1984, proposed simplifying this to 18 serogroups. Presently pathogenic strains belonging to serogroup $0: 1$. 2a,3;0:2a,3;0:3; 0:8; 0:9, 0:4, 32;0:5, 27;0:12, 25;0:13a,13b;0:19;0:20; and 0:21 have been identified. Therefore, pathogenic strains can belong to diverse serogroups. Serogroups that predominate in human illness are 0:3,0:8,0:9 and 0:5,27 (USFDA/CFSAN,2001, Brooks and Mark9 2005).

Y. enterocolitica has been isolated from rodents and domestic animals (e.g. sheep, cattle, swine, dogs and cats) and waters contaminated by them. The principal reservoir of $Y$. enterocolitica is swine. Infection with $Y$. enterocolitica is believed to be transmitted by ingestion of contaminated food (raw or incompletely cooked pork products or unpasteurized milk), by contaminated surface or well water, by direct or indirect contact with animals, by transfusion with contaminated packed red blood cells, and rarely by person to person transmission. Bottle- fed infants can be infected if their caregivers handle raw pork intestines (chitterlings). (American Academy of Pediatrics, 2006). Refrigerated foods are potential vehicles, because contamination is possible at the manufacturing site (Aulisio et al, 1982) or in the home (Jackson et al, 1999; USFDA/CFSAN 2001). Y. enterocolitica is isolated most often during the cool months of temperature climates (American Academy of Pediatrics ,2006). Prolonged assymtomatic carriage is possible. Most infected subjects are symptomatic. Death is uncommon, but the presence of $Y$. enterocolitica bacteremia is a associated with a case fatality of $34-50 \%$ (Brooks et al, 2005).The various manifestation of Yersinia infection include enterocolitis, psuedoappendicitis, reactive arthritis, erythema nodosum, septicemia, pharyngityis, dermatitis, meningitis glomerulo nephritis, pneumonia, urinary tract infections (Brooks et al, 2005, Daniel , 2006). Infections have been reported from South Africa, Zaire, Nigeria, Japan, Canada and Europe. Most have occurred in persons already in poor health (Cheesbrough, 1984). 
This study will determine the incidence and public health significance of Yersinia enterocolitica in animals in Enugu urban using cold enrichment, culture and biochemical tests as the diagnostic laboratory tests. It also studies how the organism can be contacted as well as recommendations on how the incidence can be reduced.

\section{Methodology}

A total of 106 animals, were used in this project. These animals included 50 chickens, 23 pigs and goats. These animals were obtained as shown in Table 1

Table I: Animals, Number and Sources

$\begin{array}{lll}\text { Chickens } & 50 & \text { Artisan Market in Enugu } \\ \text { Pigs } & 23 & \text { New Market in Enugu Urban } \\ \text { Goats } & 33 & \text { Artisan Market in Enugu } \\ \text { Total } & 106 & \end{array}$

The intestines of these animals were swabbed at the slaughter house and sent to the laboratory for analysis.

\section{ISOLATION AND IDENTIFICATION}

Each of the intestinal swabs was enriched in freshly prepared $0.5 \%$ potassium hydroxide $(\mathrm{KOH})$ in $0.5 \%$ sodium chloride $\mathrm{NaCI}, \mathrm{pH} 7.6$ for the period of 3 weeks at $4{ }^{\circ} \mathrm{C}$.

At the end of the period of 3 weeks, the swab was cultured on nutrient agar and incubated at $37^{\circ} \mathrm{C}$ for 24 hours and then re-cultured on MacConkey agar and also incubated at $37^{\circ} \mathrm{C}$ for 24 hours. At the end of this period, those colonies that were not mucoid and at the same time not pink were selected.

The isolates were subjected to gram staining and conventional biochemical test according to Fredrickson - Ahomaa and Korkeala, 2003.

\section{TRIPLE SUGAR IRON AGAR TEST}

The isolates were individually inoculated on, TSI agar slant. Both butt and slant inoculations were done. After inoculation, it was incubated at $37^{\circ} \mathrm{C}$.

\section{OXIDASE TEST}

The isolates were individually smeared on a filter paper. Then, the oxidase reagent $(10 \mathrm{~g} / \mathrm{L})$ was poured on the smear and observed for 10 seconds.

\section{CATALASE TEST}

About $2 \mathrm{~m} 1$ of hydrogen peroxide $\left(\mathrm{H}_{2} \mathrm{O}_{2}\right)$ was poured into a test tube. The test organism was inoculated into it and then observed.

\section{UREASE TEST}

Each test organism was inoculated into two urease agar slants. Both butt stab and slant inoculation were done. The agar slants were incubated; one at $25^{\circ} \mathrm{C}$ and the other at $37^{\circ} \mathrm{C}$.

\section{Results}

The intestines of 106 animals comprising 50 chickens, 33 goats and 23 pigs in Enugu urban between January and March 2008 were examined for the presence of Yersinia enterocolitica.

The result obtained from the tests carried on chickens shows that out of 50 chickens tested the presence of Yersinia enterocolitica was found in one being a prevalence rate of $2 \%$.

The result obtained from goats shows that out of 33 goats tested, 2 were found to be infected with Yersinia enterocolitica being prevalence rate of $6.06 \%$. The results obtained from pigs shows that out of 23 pigs tested, 2 were infected with Yersinia enterocolitica being a prevalence rate of $8.7 \%$.

Table 2: The Prevalence Rate of Yersinia enterocolitica in Animals in Enugu-Urban

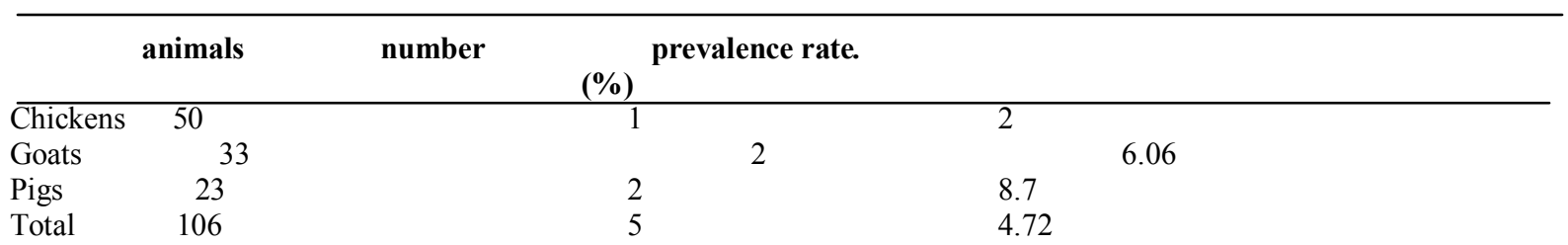




\section{Discussion}

Yersinia enterocolitica is an organism that can be found in humans, animals, water etc. It is a Psychrophile and therefore can survive in refrigerators and freezers.

In this survey on the Isolation and Public health significance of Yersinia enterocolitica in Enugu urban within the period of January to March 2008, it was observed that the prevalence rate of chickens was $2 \%$ while that of goats was $6.06 \%$ and that of pigs was $8.7 \%$. The 3 different kinds of animals combined, the prevalence rate was $4.72 \%$. This result obtained for the isolation of this microorganism from chicken though low (2\%) indicate that this animal constitutes a possible transmission path for Yersinia enterocolitica. Also, those obtained from goats and pigs were higher and the highest, respectively. Thus care should be taken when handling chitterlings of these animals.

The prevalence rate of Yersinia enterocolitica in pigs was the highest probably as a result of the contamination of the environment the pigs thrive. The prevalence rate of goats was lower than that of pigs. This could be as a result of the fact that goats do not feed on a large variety of food items as pigs. Thus reducing the number for sources of its contamination. The prevalence rate of chicken was the least. This is probably because chicken are easier to cater for. Many people have poultry farms. Chickens feed more often on industrially produced feed. Industrially produced feed is easily available, very cheap and usually, not exposed to different sources of contamination.

In a related study carried out on "the incidence of significant food borne pathogen in domestic refrigerators" the prevalence rate Yersinia enterocolitica was $0.6 \%$ (Jackson et al, 2007). This is lower than that obtained in animals in Enugu urban. This, most probably could be because some of the organisms must have died in the refrigerators because they have been exposed to a very low temperature for a very long time.

Also, in a similar study carried on cream in Brazil, the prevalence rate was $0 \%$ from the 123 industrial ice cream and cream sample (Norma and Anas, 2007). No Yersinia enterocolitic $a$ was found in industrial ice cream probably because of adequate storage temperatures, as well as proper manufacturing and handling conditions.

Bhaduri and Wesley 2006, reported prevalence rate of 3.80\% in an experiment on "The isolation and characterization of Yersinia enterocolitica from swine faeces recovered during the national animal health monitoring system swine 2000 study". The study was September 2000 to March 2001.

The presence of Yersinia enterocolitica in pigs,chickens and goats is of public health significance since contamination of food, fruits, vegetables, water etc. can be from the faeces of these animals especially when the person or persons handling them are of questionable hygiene habits.

If the untreated faeces of these animals are used as manure especially on vegetable farms, the vegetables will be contaminated and these vegetables could be a source of human outbreaks. Wesley (2007) in his literature review, Yersinia enterocolitica: an important food borne pathogen reports that consumption of dairy products (milk, ice cream), water, vegetables and pork have been linked to human outbreaks.

Again, if the person or persons handling the intestines of these animals are food handlers or day care attendees, the likelihood oftheircontaminating the food is there.

According to the study by De Boer et al, (1986) characterization of Yersinia enterocolitica and related species isolated from foods and porcine tonsils in the Netherland, Yersinia enterocolitica can be isolated from food. Therefore, if food sold in a restaurant is contaminated, this could lead to outbreak of yersiniosis. Bhaduri et al (2005) also reported a prevalence rate of $12.76 \%$ on an experiment carried on pigs from September 2000 to December 2001 titled "prevalence of pathogenic Yersinia enterocolitica strains in pigs in the united states". This prevalence rate is higher than that obtained in this study probably because of the likelihood of existence of some risk factors (such as the unhygienic condition of the environment) in the farm.

Escudero et al, (1996) reported a highest Yersinia enterocolitica recovery (26.2\%) from the faecal contents from pigs in an experiment carried on "Yersinia enterocolitica and related species in animals in San Lius, Argentina". This agrees with this present research with a highest Yersinia enterocolitica of $8.7 \%$.

This study also agrees with other literature that pig is the principal reservoir of Yersinia enterocolitica though it can be found in other animals like cattle, dogs, goats, chickens. (Brooks et al. 1998, Escudero et al 1996, Brooks and Mark 2005, Daniel , 2006).

ss Yersinia enterocolitica is of public health significance in Enugu.Someone handling the intestines of these animals could get himself infected. Such a person could be symptomatic or asymptomatic. And if the person handles public food he could get the public infected. If he is a daycare attendee, he gets the children infected and if he donates blood, he gets the recipient infected. Yersiniosis Surveillance Protocol (2008) reports that nosocomil transmission has occurred (in yersiniosis), as has transmission by transfusion of stored blood from donors who were asymptomatic or had mild gastrointestinal illness at the time of donation.

Yersinia enterocolitica, is a psychrophile. Since according to Green wood et al, (1990) in a study titled the source of Yersinia Spp. in pasteurized milk: an investigation at a dairy, that Yersinia enterocolitica can multiply at temperatures as low as $3^{\circ} \mathrm{c}$. Also, according to Jackson et al., (2005) in a study titled the incidence of 
significant food borne pathogens in domestic refrigerators, Yersinia enterocolitica can survive and grow under refrigeration or conditions of mild temperature abuse. Since Yersinia enterocolitica can grow and multiply in refrigerators if a contaminated food is put in the refrigerator, the organism multiplies in the food and could get the refrigerator contaminated and make it a source of contamination for any food item put inside it. As a result, any food, fruit, ice cream, milk etc. put in that same refrigerator get contaminated if the fruits and vegetables are not properly washed, anybody that eats them gets infected. And if the food item and meat are not properly cooked and are used to serve the public, the public gets infected and this could lead to an outbreak. Likewise, ice cream and milk put in that same refrigerator get contaminated and they could be a source of transmission of Yersiniosis to the public.Wesley (2007) in his literature Yersinia enterocolitica: an important human food borne pathogen reports that consumption of dairy products (milk, Ice cream), water, vegetables and pork have been linked to human outbreaks.

The water used in cleaning the intestines as of the aforementioned animals or even the faeces of the animals can contaminate a public source of water supply. This can cause an epidemic in a community especially if that source of water supply is the only one in that community. Also, if this organism contaminates distilled water, it can lead to a wrong chemical analysis which could mislead the public. This is because this organism can grow even in distilled water. (Highsmith et al., 1977).

\section{Recommendations}

The following recommendations will help to prevent or reduce the incidence of Yersiniosis among the populace.

1. The faeces of chickens, goats and pigs should be treated before being used as manure.

2. Symptomatic individuals should not be involved in care of young children and they should not also be allowed to prepare food or feed the children until their symptoms are resolved. Also they should not be allowed to handle public food.

3. Asymptomatic individuals with questionable hygiene habits should not be allowed to take care of young children, prepare their food or feed them. They should not also be allowed to handle public food.

4. The public should be educated on how Yersinia enterocolitica can be transmitted and how to prevent its transmission.

For instance:

1. Individuals caring for young children should not handle intestines of animals at the same time. They should wash their hands before preparing the food of these children, before feeding and after feeding. They should also wash their hands after changing diapers. They should also be educated on proper food handling.

2. Symptomatic individuals should not be involved in food handling. Asymtomatic individuals should be counseled on the importance of good hand washing and personal hygiene.

3. Fruits and vegetable should be properly washed before eating. Food and meat (especially Pork) should be cooked properly before eating.

4. Food items put in the refrigerators should be properly covered to prevent them from getting contaminated.

5. Individuals should be properly treated and also asymptomatic individuals should be excluded from handling food and caring for the young whenever they have diarrheal illness.

6. Animals should not be allowed to graze on the farm especially vegetable farm.

7. Animals should not be allowed to drink directly from the public's source of water supply for example the streams, rivers etc.

8. The slaughter houses should be sited away from residential buildings. This will prevent vectors from caring Yersinia enterocolitica from animals' faeces to people's food.

9. Wastes and faeces should be properly disposed.

10. In the case of outbreak, any common source of exposure should be identified and taken care of.

11. Symptomatic and asymptomatic individuals with mild gastro intestinal illness should not be allowed to donate blood.

\section{Conclusion}

Care should be taken when handling chitterling of animals. Hands should be washed with soap and water .Also care should be taken to ensure that the water and food these animals feed on are not contaminated. These will help to reduce the incidence of yersiniosis. 


\section{References}

[1]. Abdel-Haq N. M, Asmar B. I, Abuhammour W.M, Brown W.J. (2000). Yerisinia Enterocolitica Infection in Children. The Pediatric Infectious Disease Journal. 19(10):954-958.

[2]. American Academy of Pediatrics (2006). Summasries of Infectious Diseases: Yerisinia Pseudotuberculosis Infections. Retrievedfrom, http://aapredbook.aapublications.or/cgi/content/full/2006/1/3.151

[3]. Bhaduri, S. J. Wesley, I. V. and Bush, E. (2005). Prevalence of Pathogenic Yersinia enterocolitica Strains in Pigs in the United State. Journal of Applied and Environmental Microbiology, 71(11):117-7121.

[4]. Brooks D.C, Gregory M. J., Mark H.J. (2005). Yerisinia Enterocolitica. Retrieved from http://www.emedicine.com/med/topic2434.htm.

[5]. Cheesbrough, M. (1984). Medical Laboratory Manual for Tropical Countries. Cambridge University Press published by Tropical Health Technology, pp 58-69, 274.

[6]. Daniel, R. B. (2006). Yerisinia Enterocolitica Infection. Retrieved from http://www.emedicine.com $/ \mathrm{med} / \mathrm{topic} 2465 . h$ tm

[7]. De Boer E, Seldan W.M. and Oosteron, J. (2002). Characterization of Yerisinia Enterocolitica and related Species isolated from Foods and Porcine tonsils in the Netherlands. International Journal of Food Microbiology. 3 (4): 217-224.

[8]. Escudero, M. E. Velaquez, L. and De Guzman, A. M. S (1996). Yersinia enterocolitica and related species isolated from Animals. Journal of Food Microbiology, 13 (3):210-214.

[9]. Fredriksson-Ahomaa, M. and Korkeala, H. (2003), Low occurrence of Pathogeric Yerisinia Enterocolitica in Clinical Food and Environmental Samples: a Methodological Problem. Journal of Clinical Microbiology 16(2 ):220-229.

[10]. Jackson, V. Blair, I.S Mcdowell, D. A, Kennedy, J. and Bolton, D. J. (2005). Incidence of Significant Food borne Pathogens in Domestic Refrigerators. Journal of Food Control, 18 (4 ) 346-351.

[11]. Osman, E. (1996) Survival of virulent Yerisinia enterocolitica during the Manufacture and Storage of Turkish Feta Cheese. Intentional Journal of Food Microbiology 33(2-3):285-292.

[12]. Swetha G.P. (2009). Pediatric Yerisinia enterocolitica. Retrieved from http://emedicine.medscape.com/article/970186

[13]. USFDA/CFSAN (2001). Buttletin of the United States Food and Drug Administration/Center for Food Safety and Applied Nutrition. Yersinia Enterocolitica in: Bacteriological Analytical Manual. Retrieved from http://www.cfsan-fdagov/ebam/bam- $8 \mathrm{html}$

[14]. USFDA/CFSAN(2007). Buttletin of the United States Food and Drug Administration/Center for Food Safety and Applied Nutrition. Yerinia Enterocolitica in: Bacteriological Analytical Manual. Retrieved from http://www.cfsan-fdagov/ebam/bam-8html

[15]. Wesley, I.V. (2007). Yerisinia Enterocolitica: an Important Human Food Borne Pathogen. Natural Provisioner Magazine, p. 108 